\title{
Sensitization of Organic Photovoltaic Cells Based on Interlayer Excitation Energy Transfer
}

Musubu Ichikawa*,1,2, Eiichi Suto ${ }^{1}$, Hyeon-Gu Jeon ${ }^{1}$ and Yoshio Taniguchi ${ }^{1}$

${ }^{1}$ Functional Polymer Science Course, Faculty of Textile Science and Technology, Shinshu University, 3-15-1 Tokida, Ueda City, Nagano 386-8567, Japan

${ }^{2}$ Presto, Japan Science and Technology Agency (JST), 4-8-1 Honcho, Kawaguchi, Saitama 332-0012, Japan

(Received

)

We incorporated an additional p-type organic semiconductor layer (APL) between the anode and the phthalocyanine layer, which is an indispensable p-type semiconductor layer (IPL) in forming a $\mathrm{p} / \mathrm{n}$ junction with a fullerene $\mathrm{C}_{60}$ layer. We used two thiophene/phenylene co-oligomers as the APL. The incorporation increases the short-circuit current density $\left(\mathrm{J}_{\mathrm{SC}}\right)$ and enhances incident photon-current conversion efficiency (IPCE) over the wavelength region where the APL shows strong absorption. Combined dependence of the APL/IPL implies that Förster resonance excitation transfer is the main factor in $\mathrm{J}_{\mathrm{SC}}$ and IPCE enhancements. We demonstrate clearly that the 'positive' hole injection barrier at the interface between the APL and the IPL impacts the smooth transportation of holes to the indium-tin-oxide anode. However, the small positive hole barrier of $0.1 \mathrm{eV}$ has no noticeable influence on the fill factor of the current density vs. voltage characteristic under photoirradiation, or on those devices 
with 'negative' hole barriers.

Keywords: organic solar cells, organic photovoltaic cells, exciton transfer, excitation transfer, energy transfer, Förster resonant excitation transfer

*Corresponding author at: Functional Polymer Science Course, Faculty of Textile Science and technology, Shinshu University, 3-15-1 Tokida, Ueda City, Nagano 386-8567, Japan

Tel: +81-268-21-5498; Fax: +81-268-21-5417

Email address: musubu@shinshu-u.ac.jp 
Organic photovoltaic (PV) cells have received a lot of attention because of their potential use in applications, such as in flexible solar cells, and the low-cost production of those applications.[1] The power conversion efficiency (PCE) of organic PV cells has been increasing,[2-9] but further enhancements are required before being utilised in practical applications. To enhance the PCE, researchers must first increase the intensity of light absorption as well as broaden the available spectrum. In this context, tandem-stacking organic PV cells[7, 10, 11] is one viable option, because we can basically use four different organic semiconductors (two p-type and two n-type) in a tandem device, and each material would cover its own specific absorption spectrum.[7, 11] However, in the case of tandem devices, performance matching between each cell is very important. For example, in the case of series-connected tandem PV cells, while the open-circuit voltage $\left(\mathrm{V}_{\mathrm{OC}}\right)$ is the summation of each individual $\mathrm{V}_{\mathrm{OC}}$, the short-circuit current ( $\mathrm{J}_{\mathrm{SC}}$ ) should be limited to the lowest $\mathrm{J}_{\mathrm{SC}}$ in each cell.[7] Although balancing each cell is the key, matching the performance of each cell in the best state will be difficult. Here, we will demonstrate another method by which we can use three or more organic semiconducting materials, and in which all the materials contribute to light absorption and to the generation of charge carriers.

We introduced an additional p-type organic semiconductor layer (APL) into organic PV cells that have a single $\mathrm{p} / \mathrm{n}$ junction. In this sense, the devices are not tandem, as shown in Fig. 1(a). The APL was located between an electrode and an indispensable p-type layer (IPL), which was directly connected to an n-type layer (NL). By tuning the energy difference between the bandgap energies of both p-type materials, an APL bandgap larger than that of the IPL could be achieved, and the APL exciton 
produced by optical absorption could be transferred to the IPL, resulting in the creation of additional IPL excitons. Essentially, there is no distinguishable difference between the two types of IPL excitons produced by direct optical excitation and transfer, and consequently, the IPL exciton created by exciton transfer should also separate into charge carriers at the $\mathrm{p} / \mathrm{n}$ junction in the same way. In short, by achieving interlayer excitation energy transfer between the APL and the IPL, PV cells can be sensitized by utilizing the light absorbed by the APL.

In relation to this work, Hidel et al. have reported the sensitization of organic PV cells by incorporating an 'antenna' layer.[12] The antenna layer was positioned externally between the cathode and the anode, and was coupled with an organic PV component based on energy transfer via surface plasmon polaritons (SPPs) through a thin Ag cathode. While the 15-20-nm-thick Ag cathode successfully increased the quantum efficiency of energy transfer from the antenna, such a thin metal electrode leads to undesirable higher series resistances. The device proposed in the present study used an APL placed internally between the anode and the cathode as an antenna; it therefore requires no thin electrode with high resistivity. In addition, the SPP-based antenna required high photoluminescence (PL) efficiency,[12] whereas the APL does not require high light emissivity. This is essentially because excitation transfer from the APL to the IPL is based on a common form of nonradiative excitation energy transfer, such as Förster resonance excitation transfer (FRET) and Dexter electron exchange excitation transfer.[13, 14]

Figure 1(b) shows the schematic device structure employed in the present study, 
and Fig. 1(c) shows the chemical structures of the materials used. We used BP3T or P6T as the APL materials, and metallophthalocyanines (MPc; $\mathrm{M}=\mathrm{Cu}, \mathrm{Zn}$ and $\mathrm{Sn}$ ) as the IPL materials. APL materials should possess good hole-transporting ability, and (thiophene/phenylene) co-oligomers such as BP3T and P6T have been reported as having this ability.[15-18] The chemicals BP3T and P6T (Institute Research and Innovation), ZnPc and SnPc (Aldrich) and BCP (TCI) were purified by temperature-gradient train sublimation with $\mathrm{Ar}$ gas flow before use. We used $\mathrm{C}_{60}$, purchased from Frontier Carbon (nanom purple SUH grade) and CuPc (for the organic light-emitting device) donated by Nippon Steel Chemicals, without further purification. We prepared the devices by depositing several materials sequentially onto commercially available indium-tin-oxide (ITO) coated glass substrates, which had sheet resistance of $15 \Omega /$ square. To begin with, the substrates were washed with detergent water, pure water and 2-propanol under ultrasonification and treated with $\mathrm{O}_{2}$ plasma at a power of $50 \mathrm{~W}$ for 5 minutes. We prepared a poly(3,4-ethylenedioxythiophene) poly(styrenesulfonate) (PEDOT:PSS) layer on the substrates by spin-coating a commercially available formula (H.C. Starck, Clevios P Al 4083). We deposited several organic layers by thermal evaporation under vacuum $\left(2.0 \times 10^{-4} \mathrm{~Pa}\right)$ at a rate of $1 \AA$ s. Finally, the aluminium electrode was prepared on top of the organic layer stack by thermal evaporation in vacuum, at a rate of $10 \AA$ s. Active areas of the cells were about $6 \mathrm{~mm}^{2}$. We transferred the produced devices into a highly inert glove box $\left(\mathrm{O}_{2}\right.$ concentration $5 \mathrm{ppm}$; dew point $<-50^{\circ} \mathrm{C}$ ) with no exposure to ambient conditions, and then mounted them in a small chamber, which had a quartz window, in the glove box. A source meter (Keithley 2410) measured their current density versus voltage (J-V) curves both with and without $100-\mathrm{mW} / \mathrm{cm}^{2}$ artificial solar light irradiation of airmass 
1.5 global (AM1.5G) from a Newport Oriel solar simulator (Model 91160) with an AM1.5G filter (Newport Oriel 81088) and a light-intensity stabilizer (Newport Oriel, Model 68945). The power density of irradiation was measured with a pyroelectric optical power meter (Ophir P3). We recorded the incident photon-current conversion efficiency (IPCE) spectra of the devices with a digital multimeter (Advantest AD7461A) at zero bias, under monochromatic light irradiation from a light source (Bunko Keiki SM-25A); the intensity of the monochromatic light for each wavelength was measured with a calibrated silicon photodiode (Hamamatsu Photonics S1337-1010BQ). Surface morphologies of thin films were evaluated with a scanning probe microscope (Veeco, Dimension ICON) in tapping mode. Note that in this study we employed relatively thinner layers of MPc and $\mathrm{C}_{60}$ compared to other studies of organic PVs with MPc and/or $\mathrm{C}_{60}$, because we aimed to determine exactly how the interlayer excitation energy transfer influences organic photovoltaic devices.

Figure 2 shows the $\mathrm{J}-\mathrm{V}$ curve of the $\mathrm{BP} 3 \mathrm{~T} / \mathrm{CuPc} / \mathrm{C}_{60}$ device that had a $\mathrm{BP} 3 \mathrm{~T}$ layer as the APL. The figure also shows the curve of another PV cell $\left(\mathrm{CuPc} / \mathrm{C}_{60}\right)$ with no APL layer, as a reference. In the case of this control, we simply omitted the BP3T and the thicknesses of the other layers remained the same. As shown in the figure, placing the BP3T layer in front of the CuPc layer mainly enhanced $\mathrm{J}_{\mathrm{SC}}$ but not $\mathrm{V}_{\mathrm{OC}}$. The PCE of the $\mathrm{BP} 3 \mathrm{~T} / \mathrm{CuPc} / \mathrm{C}_{60}$ device was $0.63 \%$, much lower than that reported for $\mathrm{CuPc} / \mathrm{C}_{60}$ planar junction-based PV cells,[19] because we used thinner CuPc and $\mathrm{C}_{60}$ layers to evaluate the effectiveness of the APL. Figure 3 shows the IPCE spectra of the two devices. The IPCE increases in the shorter wavelength region where BP3T has strong absorption, as shown in the inset of the figure. This result suggests that the BP3T 
exciton produced by optical absorption is transferred to the CuPc layer and that the CuPc exciton created by the transfer was converted to charge carriers at the interface of $\mathrm{CuPc} / \mathrm{C}_{60}$. It is possible that the sensitization resulted from a direct contact between BP3T and $\mathrm{C}_{60}$, through defects in the CuPc layer because of the thinness of the layer; we will discuss this point later. Note that another increase occurs around $600 \mathrm{~nm}$, which may not have been caused by the transfer. We are not sure why this other increase occurred, but we believe that the BP3T layer can prevent the CuPc exciton, produced by optical absorption, from quenching by the PEDOT:PSS layer.

Figure 4 shows the IPCE spectrum of another cell using BP3T, in which we replaced CuPc with SnPc as the IPL (device structure abbreviated as BP3T/SnPc/ $\mathrm{C}_{60}$ ). We observed no noticeable enhancement of IPCE due to light absorption by BP3T as compared to its reference device that contained no BP3T layer $\left(\mathrm{SnPc} / \mathrm{C}_{60}\right)$. These devices include two heterojunctions: one is IPL/C 60 and the other is APL/IPL. We believe that charge separation occurred in the case of the APL excitons at the APL/IPL interfaces possibly because the lowest unoccupied molecular orbital (LUMO) levels of BP3T and P6T are higher than those of the phthalocyanine (Pc) derivatives used.[20] If the sensitization by the APL occurred because of charge separation at the APL/IPL interface, then the efficiency of charge separation in the case of the Marcus normal region increases with the increasing negativity of the LUMO level of SnPc should lead to more efficient.[21] However, no noticeable enhancement of IPCE due to BP3T occurred for the BP3T/SnPc device, as shown in Fig. 4. Consequently, the sensitization mechanism by the APL differs from the double exciton dissociation at both sides of a Pc layer reported by Sista et al.[22] and Zhang et al.[23] There could essentially be a 
difficulty with this double exciton-dissociation in that the layer, both sides of which would contribute to exciton dissociations, would need to have sufficient charge-carrier mobilities for both electron and hole.

On the other hand, a remarkable enhancement appeared from $400 \mathrm{~nm}$ to $550 \mathrm{~nm}$ in the case of the device with the $\mathrm{P} 6 \mathrm{~T} / \mathrm{SnPc} / \mathrm{C}_{60}$ structure. As shown in the inset of Fig. 4, the surfaces of both the BP3T and P6T layers were slightly rough as a result of the high crystalline nature of these materials, and the root-mean-square roughness (RMS) of the layers, each $40 \mathrm{~nm}$ thick, was $8.1 \mathrm{~nm}$ for BP3T and $7.6 \mathrm{~nm}$ for P6T. It is possible that these rough surfaces induce a fairly direct contact between the APL and $\mathrm{C}_{60}$, through defects in the 10-nm-thick IPL. However, no IPCE enhancement in the $\mathrm{BP} 3 \mathrm{~T} / \mathrm{SnPc} / \mathrm{C}_{60}$ system and the remarkable IPCE improvements in both BP3T/CuPc/ $\mathrm{C}_{60}$ and $\mathrm{P} 6 \mathrm{~T} / \mathrm{SnPc} / \mathrm{C}_{60}$ systems, greatly refute that the APL mainly touches the $\mathrm{C}_{60}$ layer directly and causes the sensitizations when introducing the APLs.

The sensitized spectral region of the $\mathrm{P} 6 \mathrm{~T} / \mathrm{SnPc} / \mathrm{C}_{60}$ device increased in wavelength as compared to the BP3T/CuPc/ 600 device, as shown in Figs. 3 and 4 . This change occurred because P6T shows absorption in a larger wavelength region than BP3T, as shown in the inset of Fig. 3. Furthermore, P6T displays photoluminescence (PL) in a longer wavelength region than BP3T, and SnPc preferentially absorbs longer wavelength light than CuPc. Consequently, the overlap integral becomes large in the case of combined P6T and SnPc, where the overlap integral indicates a spectral overlap between the photoluminescence of an excitation energy donor and the absorption of an excitation energy acceptor. In addition, we observed clear sensitization due to P6T as 
the APL in the case of the $\mathrm{P} 6 \mathrm{~T} / \mathrm{CuPc} / \mathrm{C}_{60}$ cells, where there was a sufficient spectral overlap between the PL of P6T and the optical absorption of CuPc. From these results, we believe that it was probably the following scheme that increased IPCE: the BP3T or P6T layer in front of the Pc derivative layer absorbed the corresponding light, resulting in the formation of its exciton; the exciton was transferred to the back Pc derivative layer; and finally, the Pc exciton separated both the charge carriers at the Pc- $\mathrm{C}_{60}$ interface. The abovementioned spectral overlap dependence suggests that the mechanism of excitation transfer from front layer to back layer may be FRET. We are planning further studies to shed light on the mechanism of excitation transfer occurring in the cells; its quantum efficiency, including the determination of Förster radii between those thiophene derivatives and the phthalocyanines; and the intrinsic exciton diffusion lengths of those thiophenes.

It is worth noting that, while finalizing the present manuscript, Hong et al. reported an organic PV device with a pentacene/ZnPc/ $\mathrm{C}_{60}$ multi-heterojunction.[24] The concept described in that literature is very similar to that in the present study, and they utilized pentacene as a sensitizer for a $\mathrm{ZnPc} / \mathrm{C}_{60}$ heterojunction. They concluded that endothermic triplet energy transfer occurred between pentacene and ZnPc. Although both singlet and triplet energy transfers have the potential to sensitize by means of excitation transfer, what multiplicity of exciton is more advantageous in this sensitization needs to be discussed in the next stages of research. This is because a compromise between exciton lifetime and the reachable range of excitation transfer for the two types of excitons-singlet and triplet should be considered. In any case, an increase in both $\mathrm{J}_{\mathrm{SC}}$ and IPCE, and, moreover, an unchanged $\mathrm{V}_{\mathrm{OC}}$ have been clearly 
observed in the present study as a result of singlet-exciton transfer between APL-IPL as discussed above.

Finally, we discuss the case where the hole generated at the $\mathrm{p} / \mathrm{n}\left(\mathrm{Pc} / \mathrm{C}_{60}\right)$ junction is required to pass over the additional IPL-APL interface. Depending on the combination of materials, there is a hole injection barrier at the interface, and then the barrier probably affects hole transportation from the $\mathrm{p} / \mathrm{n}$ junction to the electrode. We estimated the series resistance of a device from the inverse of the slope of the tangential line (TL) of the $\mathrm{J}-\mathrm{V}$ curve at $\mathrm{V}_{\mathrm{OC}}$, as shown in Fig. 2. Figure 5 summarizes the resistances of six devices with an APL, where the injection barrier of holes $\left(\Delta \mathrm{E}_{\mathrm{H}}\right)$ is defined as the energy difference between the highest occupied molecular orbital (HOMO) levels of the APL and the IPL.[20] Negative $\Delta \mathrm{E}_{\mathrm{H}}$ means that the hole is injected endothermically from the IPL to the APL. The resistance decreases gradually with decreasing $\Delta \mathrm{E}_{\mathrm{H}}$, but increases rapidly when $\Delta \mathrm{E}_{\mathrm{H}}$ is positive. However, the fill factors (FF) of all the cells prepared are about 0.5 whether with or without the APL. In short, normalized FF for each device with the APL is almost 1 as shown in Fig. 5, where the normalized FF is defined as the ratio of FFs between PV cells using a certain Pc with and without each APL. This means that neither the large exothermic hole injection at the P6T/SnPc interface nor the small 'positive' hole injection barrier of $0.1 \mathrm{eV}$ at the BP3T/ZnPc interface influenced the charge collection through the APL/IPL interface. Furthermore, as one can see from the fairly good FFs of the cells, the entire devices show clear rectifying characteristics whether with or without APLs. Consequently, this concept would probably work well when we adjust the difference of the bandgaps of both the APL and the IPL and align their HOMO levels, appropriately. 
In conclusion, we have demonstrated the sensitization of organic PV cells with a single $\mathrm{p} / \mathrm{n}$ junction based on interlayer excitation energy transfer. We incorporated an additional p-type semiconducting organic material layer (APL) between the PEDOT:PSS layer and a Pc layer, indispensable p-type semiconductor layer (IPL), to form a $\mathrm{p} / \mathrm{n}$ junction with the $\mathrm{C}_{60}$ layer for generating charge carriers from excitons. Incorporating the APL led to sensitization of the IPCE, and the sensitized wavelength regions changed depending on the absorption spectra of the APL. The combination dependence of APL/IPL suggests that IPCE enhancements mainly result from Förster resonance excitation transfer (FRET). We demonstrated clearly that the 'positive' hole injection barrier at the APL-IPL interface has an impact on the smooth transportation of holes to the anode (indium-tin-oxide). The PV cells demonstrated here showed an insufficient PCE of below 1\%, but we believe that this approach will lead to further improvements in the PCE of organic PV cells in the future. We are now working to incorporate more APLs for more efficient light collection with cascading excitation transfer toward the IPL.

This work was partly supported by CLUSTER (second stage) of the Ministry of Education, Culture, Sports, Science and Technology (MEXT), Japan.

\section{References}

[1] S. R. Forrest, Nature 428 (2004) 911.

[2] C. W. Tang, Appl. Phys. Lett. 48 (1986) 183.

[3] P. Peumans, S. R. Forrest, Appl. Phys. Lett. 79 (2001) 126.

[4] C. J. Brabec, N. S. Sariciftci, J. C. Hummelen, Adv. Funct. Mater. 11 (2001) 15. 
[5] J. Xue, S. Uchida, B. P. Rand, S. R. Forrest, Appl. Phys. Lett. 84 (2004) 3013.

[6] A. Hayakawa, O. Yoshikawa, T. Fujieda, K. Uehara,S. Yoshikawa, Appl. Phys. Lett. 90 (2007) 163517.

[7] J. Y. Kim, K. Lee, N. E. Coates, D. Moses, T.-Q. Nguyen, M. Dante, A. J. Heeger, Science 317 (2007) 222.

[8] K. Sakai, M. Hiramoto, Mol. Cryst. Liq. Cryst. 491 (2008) 284

[9] S. Wang, E. I. Mayo, M. D. Perez, L. Griffe, G. Wei, P. I. Djurovich, S. R.

Forrest, M. E. Thompson, Appl. Phys. Lett. 94 (2009) 233304.

[10] M. Hiramoto, M. Suezaki, M. Yokoyama, Chem. Lett. 19 (1990) 327.

[11] J. Xue, S. Uchida, B. P. Rand, S. R. Forrest, Appl. Phys. Lett. 85 (2004) 5757.

[12] T. D. Heidel, J. K. Mapel, M. Singh, K. Celebi, M. A. Baldo, Appl. Phys. Lett. 91 (2007) 093506.

[13] T. Förster, Discuss. Faraday Soc. 27 (1959) 7.

[14] D. L. Dexter, J. Chem. Phys. 21 (1953) 836.

[15] M. Ichikawa, H. Yanagi, Y. Shimizu, S. Hotta, N. Suganuma, T. Koyama,Y. Taniguchi, Adv. Mater. 14 (2002) 1272.

[16] H. Yanagi, Y. Araki, T. Ohara, S. Hotta, M. Ichikawa,Y. Taniguchi, Adv. Funct. Mater. 13 (2003) 767.

[17] K. Nakamura, M. Ichikawa, R. Fushiki, T. Kamikawa, M. Inoue, T. Koyama,Y. Taniguchi*, Jpn J. Appl. Phys. 43 (2004) L100.

[18] S. Hotta, T. Katagiri, T. Yamao, K. Shimizu, H. Yanagi, M. Ichikawa,Y. Taniguchi, Int J Polym Mater 57 (2008) 515.

[19] J. Xue, B. P. Rand, S. Uchida,S. R. Forrest, Adv. Mater. 17 (2005) 66.

[20] Lowest unoccupied molecular orbital (LUMO)/highest occupied molecular 
orbital (HOMO) levels of the materials used in this study: -2.8/-5.1 (BP3T), -2.6/-4.7 (P6T), -3.5/-5.2 (CuPc), -3.4/-5.0 (ZnPc), -4.2/-5.7 (SnPc) and -4.5/-6.2 ( $\left.\mathrm{C}_{60}\right)$. Unit of the levels is eV. We estimated HOMO and LUMO levels for BP3T and P6T from the ionization potential and the optical absorption edges of each thin-film. The others were from the literature.

[21] R. A. Marcus, Rev. Mod. Phys. 65 (1993) 599.

[22] S. Sista, Y. Yao, Y. Yang, M. L. Tang, Z. Bao, Appl. Phys. Lett. 91 (2007) 223508.

[23] G. Zhang, W. Li, B. Chu, L. Chen, F. Yan, J. Zhu, Y. Chen,C. S. Lee, Appl. Phys. Lett. 94 (2009) 143302.

[24] Z. R. Hong, R. Lessmann, B. Maennig, Q. Huang, K. Harada, M. Riede, K. Leo, J. Appl. Phys. 106 (2009) 064511.

\section{Figure and Table Captions}

Figure 1. (a) Conceptual energy diagram of PV cell proposed in this study, (b) sectional cell structure and (c) structures of chemicals and their abbreviations used in this study.

Figure 2. J-V curves of PV cells with or without BP3T as the APL under 1 sun irradiation. TL: tangential line at $\mathrm{V}_{\mathrm{OC}}$.

Figure 3. IPCE spectra of PV cells with and without BP3T as the APL. Inset: absorption spectra of thin films of BP3T, P6T and SnPc, and PL spectra of BP3T and P6T thin films. Thin films were deposited on quartz plates by thermal evaporation. 
Figure 4. IPCE spectra of SnPc-based PV cells incorporating BP3T (blue solid triangles), P6T (red solid squares) as the APLs, and a control cell (black open circles). The inset shows the surface morphologies of 40-nm-thick BP3T (a) and P6T (b) layers deposited on a 40-nm-thick PEDOT:PSS layer. The field size of both images are the same (1 $\mu \mathrm{m} \times 1 \mu \mathrm{m})$ and the colour bar next to image (b) is the height scale: full scales of the bar for (a) and (b) are $58.5 \mathrm{~nm}$ and $48.7 \mathrm{~nm}$, respectively.

Figure 5. Hole injection barrier $\Delta \mathrm{E}_{\mathrm{H}}$ dependence of $\mathrm{R}_{\mathrm{S}}$ and normalized FF (see text) of all the devices with APLs obtained under photoirradiation. The thick grey line is a guide for the eye. $\Delta \mathrm{E}_{\mathrm{H}}$ was calculated from the difference in HOMO levels between the APL and the IPL for each cell. 

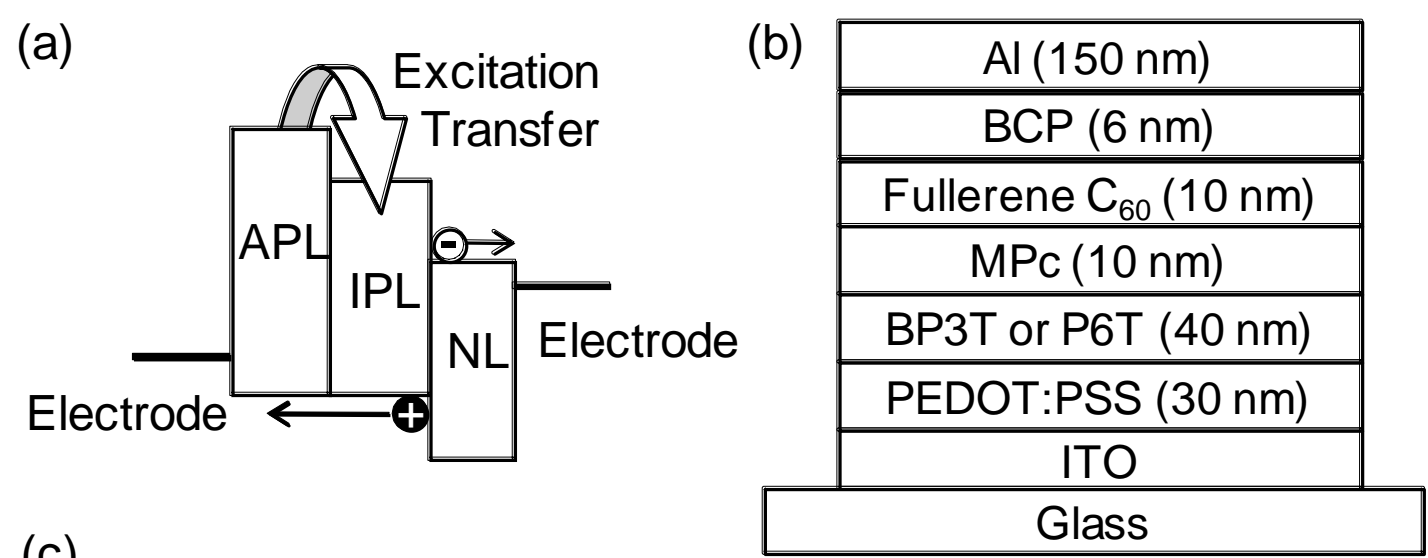

(c)
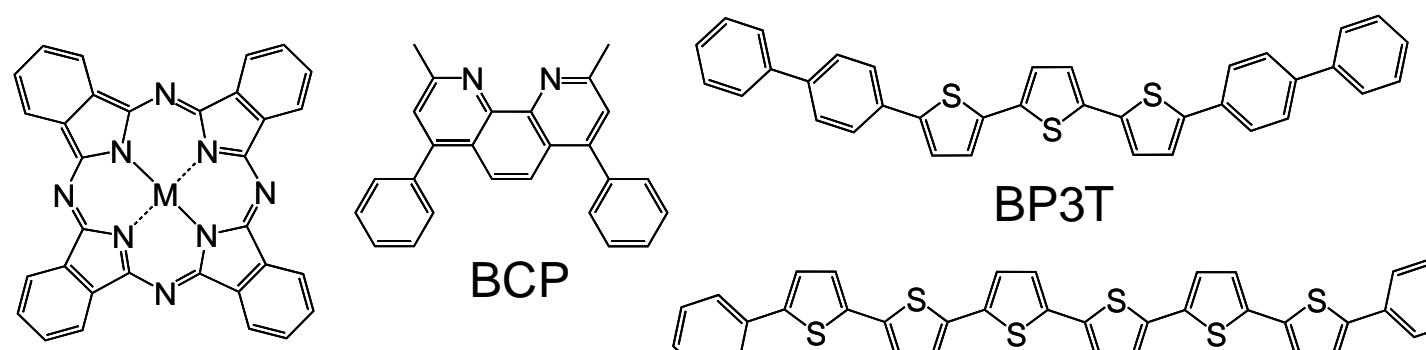

$\operatorname{MPc}(\mathrm{M}=\mathrm{Cu}, \mathrm{Zn}, \mathrm{Sn})$

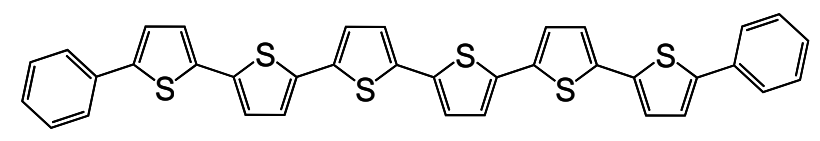

P6T

Figure 1. (a) Conceptual energy diagram of PV cell proposed in this study, (b) sectional cell structure and (c) structures of chemicals and their abbreviations used in this study. 


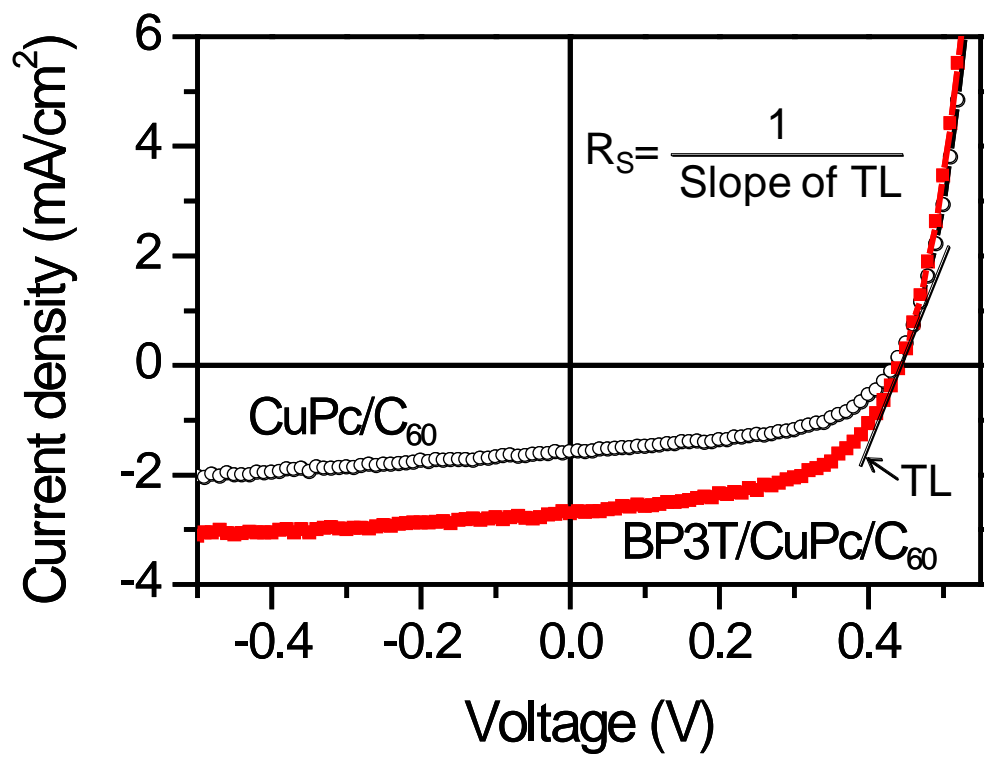

Figure 2. J-V curves of PV cells with or without BP3T as the APL under 1 sun irradiation. TL: tangential line at $\mathrm{V}_{\text {OC. }}$ 


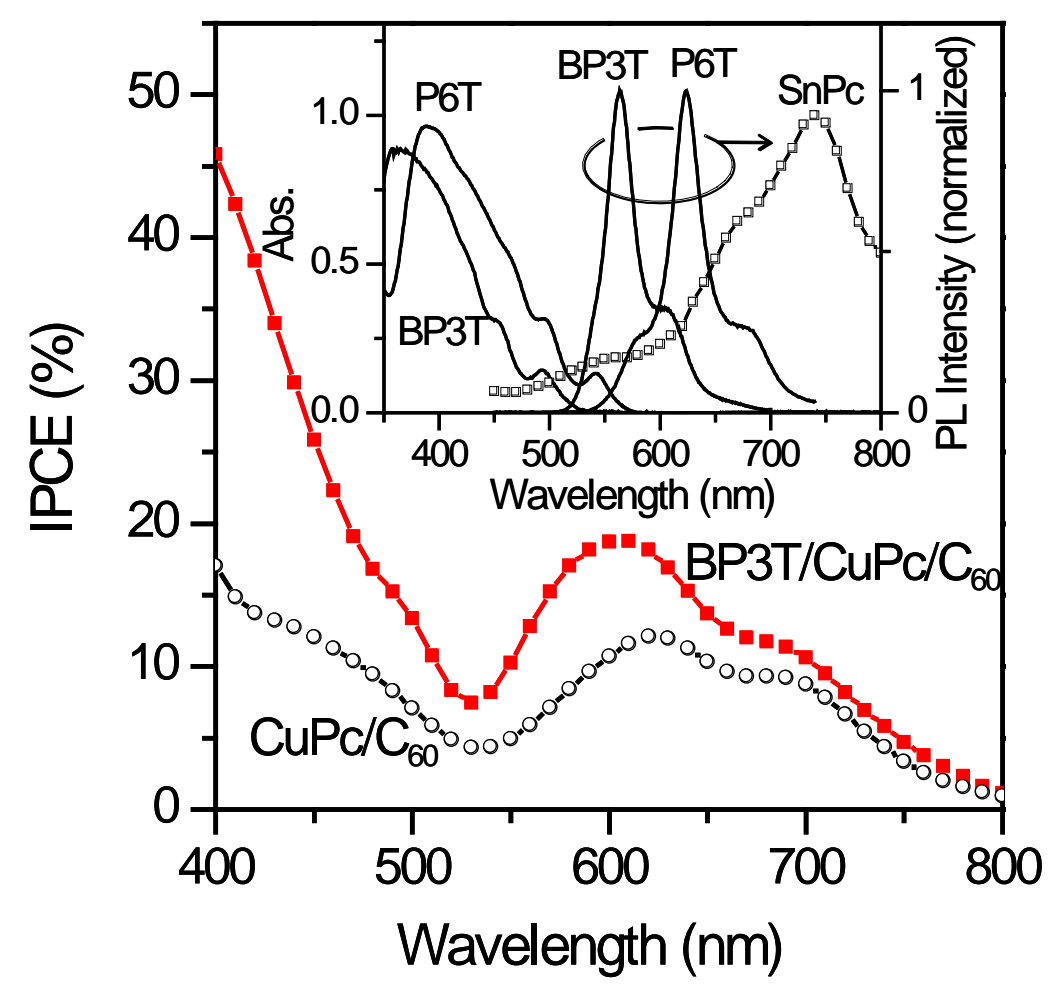

Figure 3. IPCE spectra of PV cells with and without BP3T as the APL. Inset: absorption spectra of thin films of BP3T, P6T and SnPc, and PL spectra of BP3T and P6T thin films. Thin films were deposited on quartz plates by thermal evaporation. 

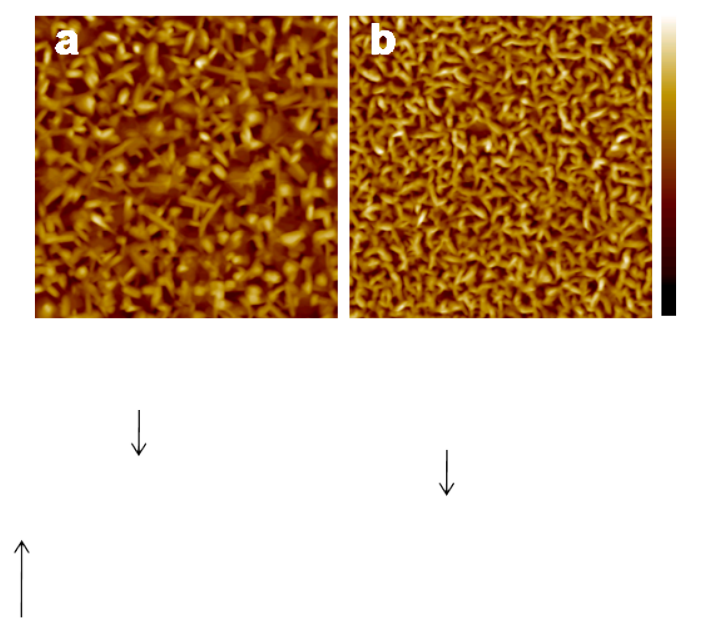

Figure 4. IPCE spectra of SnPc based PV cells incorporating BP3T (blue solid triangles), P6T (red solid squares) as the APLs and a control cell (black open circles). The inset shows the surface morphologies of 40-nm-thick BP3T (a) and P6T (b) layers deposited on a 40-nm-thick PEDOT:PSS layer. The field size of both images are the same $(1 \mu \mathrm{m} \times 1 \mu \mathrm{m})$ and the colour bar next to image (b) is the height scale: full scales of the bar for (a) and (b) are $58.5 \mathrm{~nm}$ and $48.7 \mathrm{~nm}$, respectively. 


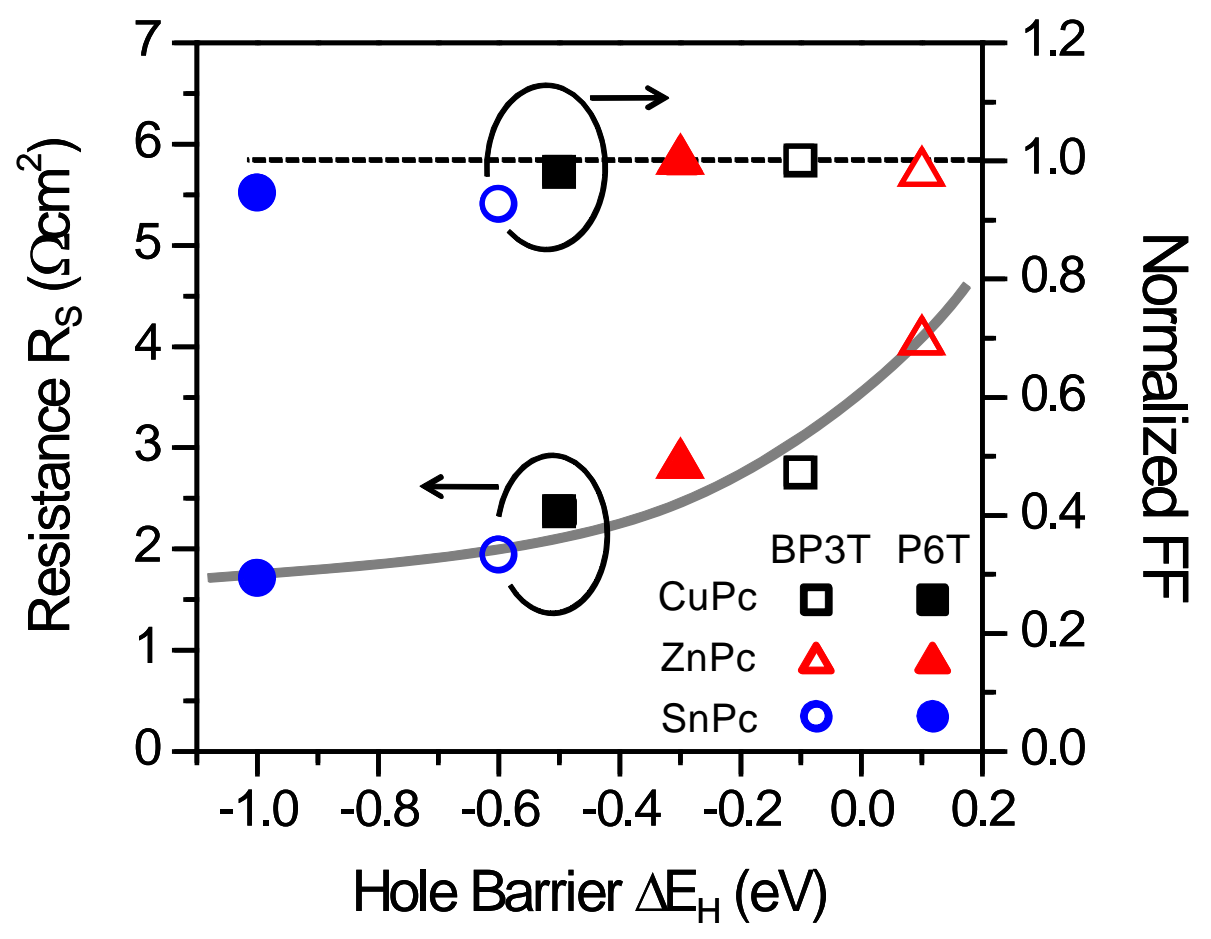

Figure 5. Hole injection barrier $\Delta \mathrm{E}_{\mathrm{H}}$ dependence of $\mathrm{R}_{\mathrm{S}}$ and normalized $\mathrm{FF}$ (see text) of all the devices with APLs obtained under photoirradiation. The thick grey line is a guide for the eye. $\Delta \mathrm{E}_{\mathrm{H}}$ was calculated from the difference in HOMO levels between the APL and the IPL for each cell. 